\title{
A study of the early morphological changes initiated in the uterine luminal epithelium by substances (oil and carrageenan) which induce the decidual cell reaction in mice
}

\author{
C. A. Finn, M. D. Pope and S. R. Milligan* \\ Department of Veterinary Preclinical Sciences, University of Liverpool, P.O. Box 147, \\ Brownlow Hill, Liverpool L69 3BX, UK; and *Department of Physiology, King's College, Strand, \\ London $W C 2 R 2 L S, U K$
}

\begin{abstract}
Summary. Oil, carrageenan or saline were injected into the uteri of ovariectomized mice treated with hormones on schedules which would sensitize, partly sensitize or not sensitize the uterus to an intraluminal decidual stimulus. The uterine epithelium was examined histologically at various times over the succeeding $5 \mathrm{~h}$. Saline did not produce any morphological change whereas almost immediately after the injection of oil or carrageenan epithelial cell death was apparent in the uterus, regardless of hormone treatment. Within $45 \mathrm{~min}$ the dead cells had been removed and the epithelium was reestablished. Oil droplets were still present in the uterus after $5 \mathrm{~h}$ and these were able to stimulate a decidual reaction in partly sensitized animals when oestrogen was administered 18-44 $\mathrm{h}$ after the oil instillation, well after the re-establishment of the epithelium. It is suggested that the early transient cell death in the uterine epithelium is not responsible for triggering the decidual reaction but that it is the contact of the oil droplet with an intact epithelium which triggers the response when the hormonal conditions so allow.
\end{abstract}

Keywords: decidualization; uterus; implantation; endometrium; mouse

\section{Introduction}

A problem of fundamental importance in the understanding of implantation is how the stromal cells of the endometrium are stimulated to differentiate into decidual cells. In women decidualization can take place spontaneously in response to ovarian hormones, but in most deciduate species it is necessary for a stimulus to be applied to the hormonally prepared uterus to initiate a decidual reaction. Normally the blastocyst provides this stimulus. Differentiation can be initiated experimentally in the hormonally sensitized animals, however, by applying artificial stimuli to the uterus. This was shown originally by Loeb (1908) who traumatized the uteri of guinea-pigs at the appropriate stage of the cycle and induced a decidual cell reaction (DCR). Subsequently, other stimuli applied to the lumen of the uterus have been shown to be good inducers of the DCR. In mice, intraluminal injections of oil or a solution of carrageenan are potent deciduogenic stimuli (Finn \& Keen, 1963) and oil is now widely used to initiate a decidual reaction. The injection of normal saline does not initiate a decidual reaction, except sometimes at the site of injection. The mechanism whereby oil or carrageenan or trauma or indeed the blastocyst induce a decidual reaction is unknown.

An important question which needs to be settled is the extent to which the various stimuli operate through similar mechanisms. It is tempting to assume that all such stimuli act through 
some common final pathway. It is uncertain, however, whether the initial responses to the stimuli share any similarities. In the mouse differences are certainly apparent in the endocrine requirements for traumatic as opposed to intraluminal stimuli: trauma induces decidualization after treatment with progesterone alone, whilst intraluminal stimuli (e.g. oil or carrageenan) resemble the blastocyst in requiring small amounts of oestrogen as well (Finn, 1965). Such observations have led to the concept that in rats and mice trauma may bypass initial oestrogen-sensitive steps that are normally initiated by intraluminal stimuli.

Lundkvist \& Nilsson (1982) examined the histological responses to intraluminal instillation of oil and found epithelial cell damage within $15 \mathrm{~min}$ after instillation in the uteri of sensitized rats but not in the uteri of non-sensitized animals. From these results they concluded that these agents acted as a traumatic stimulus to the luminal epithelium. The present study examined the effects of oil, carrageenan or saline on the morphology of the mouse uterine luminal epithelium in relation to their deciduogenic potential under various hormonal conditions.

\section{Materials and Methods}

Mature female mice were ovariectomized under alphaxalone and alphadolone acetate (Saffan, Glaxovet Ltd, Harefield, Uxbridge, Middlesex, UK) anaesthesia. All animals were rested for at least 1 week before start of hormone treatment. Hormones were administered by subcutaneous injection dissolved in arachis oil $(0.05 \mathrm{ml})$. Mice were allocated to the following hormone treatments.

Treatment 1 . Two daily injections of $100 \mathrm{ng}$ oestradiol followed by 3 days rest then daily injections of $500 \mu \mathrm{g}$ progesterone plus $10 \mathrm{ng}$ oestradiol. This produces a condition of full sensitivity to an intraluminal decidual stimulus on the 3rd day of the progesterone and oestradiol treatment (Finn \& Martin, 1969).

Treatment 2. Two daily injections of $100 \mathrm{ng}$ oestradiol followed by 3 days without hormone treatment then daily injections of $500 \mu \mathrm{g}$ progesterone. This produces on the $3 \mathrm{rd}$ day of progesterone treatment a partly sensitized condition, equivalent to that found in delayed implantation. Oil will not induce a DCR under these conditions but overt trauma, such as crushing of the uterus, will do so.

Treatment 3. Two daily injections of $100 \mathrm{ng}$ oestradiol.

\section{Decidual stimuli}

The mice were anaesthetized (Saffan) and $0.015 \mathrm{ml}$ of $0.9 \%(\mathrm{w} / \mathrm{v}) \mathrm{NaCl}$, arachis oil or a $1 \%$ solution of carrageenan (Sigma, P.O. Box 14508, St Louis, MO 63178, USA) was injected into the uterine lumen through the utero-tubal junction. In Treatment 3, this was carried out 6 days after the 2 nd oestradiol injection and in Treatments 1 and 2 on the 3 rd day of progesterone or progesterone plus oestradiol treatment, $4-6 \mathrm{~h}$ after the hormone administration.

At various times after the intrauterine instillation the animals were killed and the uteri fixed. In most cases the animals were killed by cervical dislocation and the uteri fixed by immersion in Karnovsky's (modified) fixative. In a few cases the animals were anaesthetized and the fixative was infused through the aorta. The uteri were embedded in the usual way in plastic and sections cut and examined with the light or electron microscope.

The first experiment revealed that many of the early morphological responses to oil disappeared after a few hours, yet oil was retained in the uterine lumen for many hours after instillation. A second experiment was therefore undertaken to investigate whether the uterus would still respond with decidualization when the sensitizing injection of oestradiol was delayed until after the early morphological responses to oil instillation had disappeared.

\section{Results}

\section{Experiment 1: morphology of the endometrium at various times after intrauterine instillation}

Saline. There was no disruption of the luminal epithelium, histological changes in the epithelial cells, or cellular debris within the uterine lumen, at any time in Treatment $1(\mathrm{~N}=7,5$ and 7 , at $0 \mathrm{~min}, 45 \mathrm{~min}$ and $2.5 \mathrm{~h}$ after instillation, respectively). In view of the lack of response in these animals, the effects of saline were only examined in Treatments 2 and 3 at $2.5 \mathrm{~h}$ after instillation: in all cases the epithelium was intact and there were no signs of cellular debris in the lumen. 

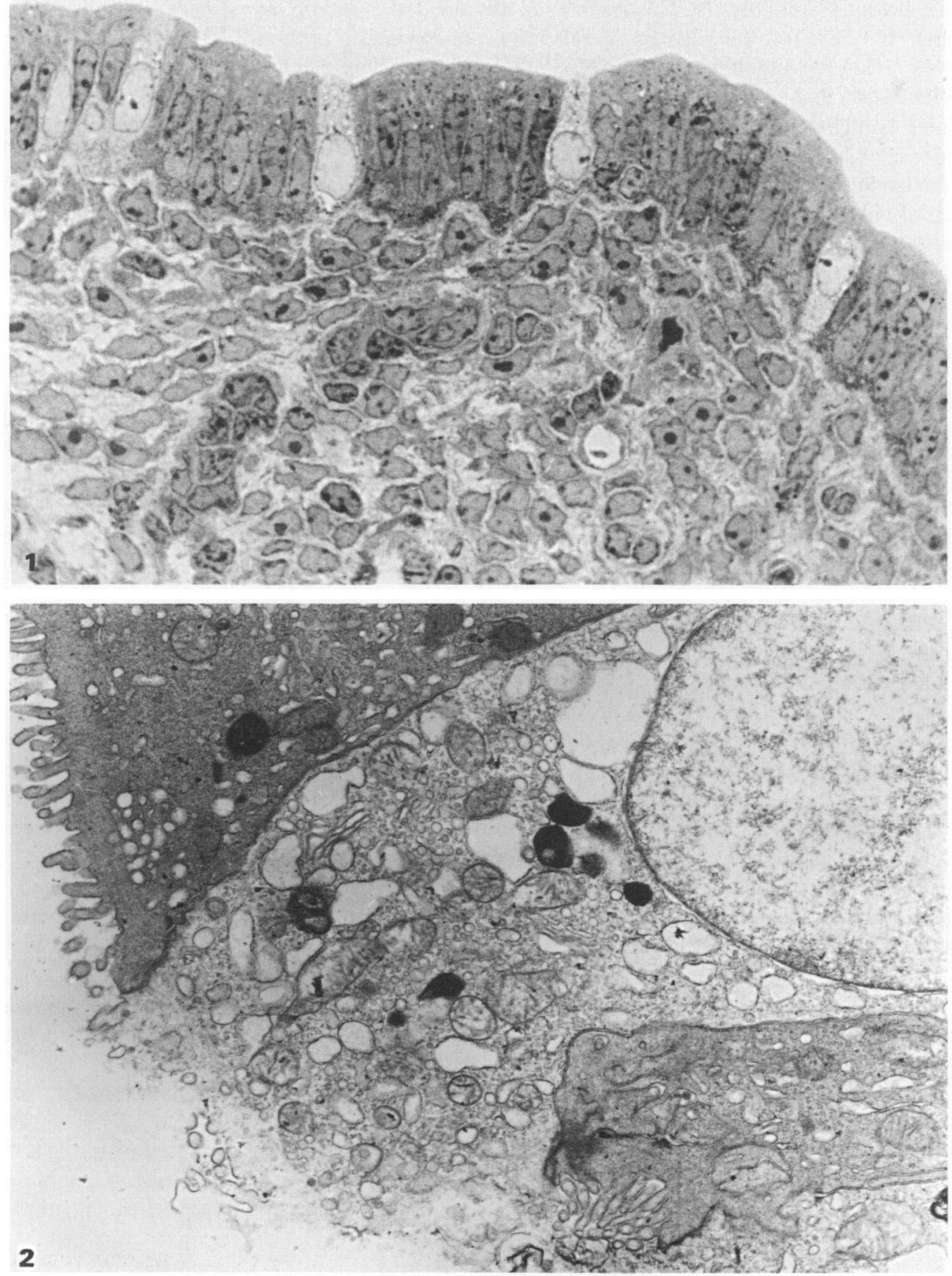

Fig. 1. Light micrograph of part of the uterine luminal epithelium and stroma from a mouse killed within $1 \mathrm{~min}$ of the intrauterine instillation of oil. Several pale cells can be seen in the epithelium. $\times 250$.

Fig. 2. Electron micrograph showing the luminal end of one of the pale epithelial cells depicted in Fig. 1. $\times 12000$. 
Oil. Oil instillation induced gross histological changes in the uterus in all treatment groups.

In uteri fixed by immersion immediately after oil instillation $(\mathrm{N}=8,6$ and 6 , for Treatments 1 , 2 and 3, respectively), the luminal epithelium showed the presence of variable numbers of very lightly staining cells (Fig. 1). The numbers in any one cross-section varied between approximately 10 and $70 \%$. Electron microscopy revealed that the chromatin in the enlarged nuclei of these cells was more dispersed than in adjacent cells, although the nuclear membrane appeared intact (Fig. 2). Mitochondria and coated vesicles were enlarged, the apical cell membrane was disrupted, with the cellular contents apparently being lost to the lumen (Fig. 3). There was no difference in the incidence of these cells in the three treatments.

To examine the time course of these early responses to oil, a few animals treated with progesterone plus oestradiol (Treatment 1) were rapidly perfused with fixative 3,6 or 10 min after oil instillation. No changes were seen using light microscopy at $3 \mathrm{~min}$. After $6 \mathrm{~min}$, the nuclei of not more than $10 \%$ luminal epithelial cells were enlarged and granulated, and the number of cells showing changes had increased further by $10 \mathrm{~min}$.

By 25-45 min after instillation, the majority of animals (8/10 and $7 / 10$, respectively) in Treatments 1 and 2 had cellular debris and oil droplets in the lumen. The lining epithelium was intact and very few pale cells were found. Only $2 / 8$ animals from Treatment 3 showed cellular debris in the uterine lumen.

At $2.5 \mathrm{~h}$ after instillation, 11/16 animals in Treatment, 1, 2/11 in Treatment 2 and $2 / 12$ in Treatment 3 showed cellular debris in the lumen. The numbers showing oil droplets were 10/16, $6 / 11$ and $3 / 12$, respectively. Pale cells were no longer present and the epithelium appeared intact.

At $5 \mathrm{~h}$, cellular debris was present in the lumen of $3 / 3$ animals in each of Treatments 1 and 2 , and oil droplets in $2 / 3$. By $10 \mathrm{~h}$, cellular debris was present in only $2 / 8$ animals in Treatment 1 , and $0 / 8$ in Treatment 2 . Oil was still present in the uteri of $5 / 8$ animals in each of these treatment groups. No pale cells were present.

Carrageenan. Carrageenan instillation induced gross histological changes in the uterus in all treatments, but the response was very different from that seen after oil injection.

In all the uteri fixed by immersion immediately after carrageenan instillation $(N=8,6$ and 6 , for Treatments 1,2 and 3, respectively), the luminal epithelium showed the presence of a variable number (between 10 and $70 \%$ ) of very lightly staining cells. Electron microscopy, however, revealed that these cells were very different from those seen after oil instillation (Figs 4 \& 5). The lightly staining cells induced by carrageenan were considerably larger, with very enlarged, pale nuclei. Cellular contents were sparse apart from a very few dilated profiles of endoplasmic reticulum and occasional mitochondria. Frequently the cells were found deep in the epithelium, surrounded by cells of normal appearance. Where the cells were on the luminal surface, the apical membrane was intact and there was no evidence of discharge of contents int the lumen. Cellular debris was never found in the lumen. Carrageenan also induced extensive dilatation of the intercellular spaces, giving the epithelium a lacelike appearance. Such a response was never seen after oil instillation.

At 25 min after instillation, pale cells were still found in the luminal epithelium of all animals in Treatments 1 and 2. The lacy appearance of the epithelium due to the greatly enlarged intercellular spaces was still very apparent. No cellular debris was present in the uterine lumen.

At $2.5 \mathrm{~h}$ after carrageenan instillation, a few (10-20\%) lightly staining cells were present in only $2 / 9$ animals in Treatment 1 and were not seen in any of the animals in Treatment $2(\mathrm{~N}=4)$. There was no sign of the previously enlarged intercellular spaces, or of cellular debris in the uterine lumen. A further 4 animals from Treatment 1 were examined $5 \mathrm{~h}$ after instillation of carrageenan. None of the uteri showed the lightly staining cells, although in this treatment group leucocytes and red blood cells were seen in the uterine lumen.

Experiment 2: uterine decidual response after oil instillation but with delayed oestradiol sensitization

In the initial experiment, oil was instilled into both uterine horns of partly sensitized animals (Treatment 2 above) and the mice were injected with $20 \mathrm{ng}$ oestradiol-17 $\beta$ at $10(\mathrm{~N}=5)$ or $16 \mathrm{~h}$ 

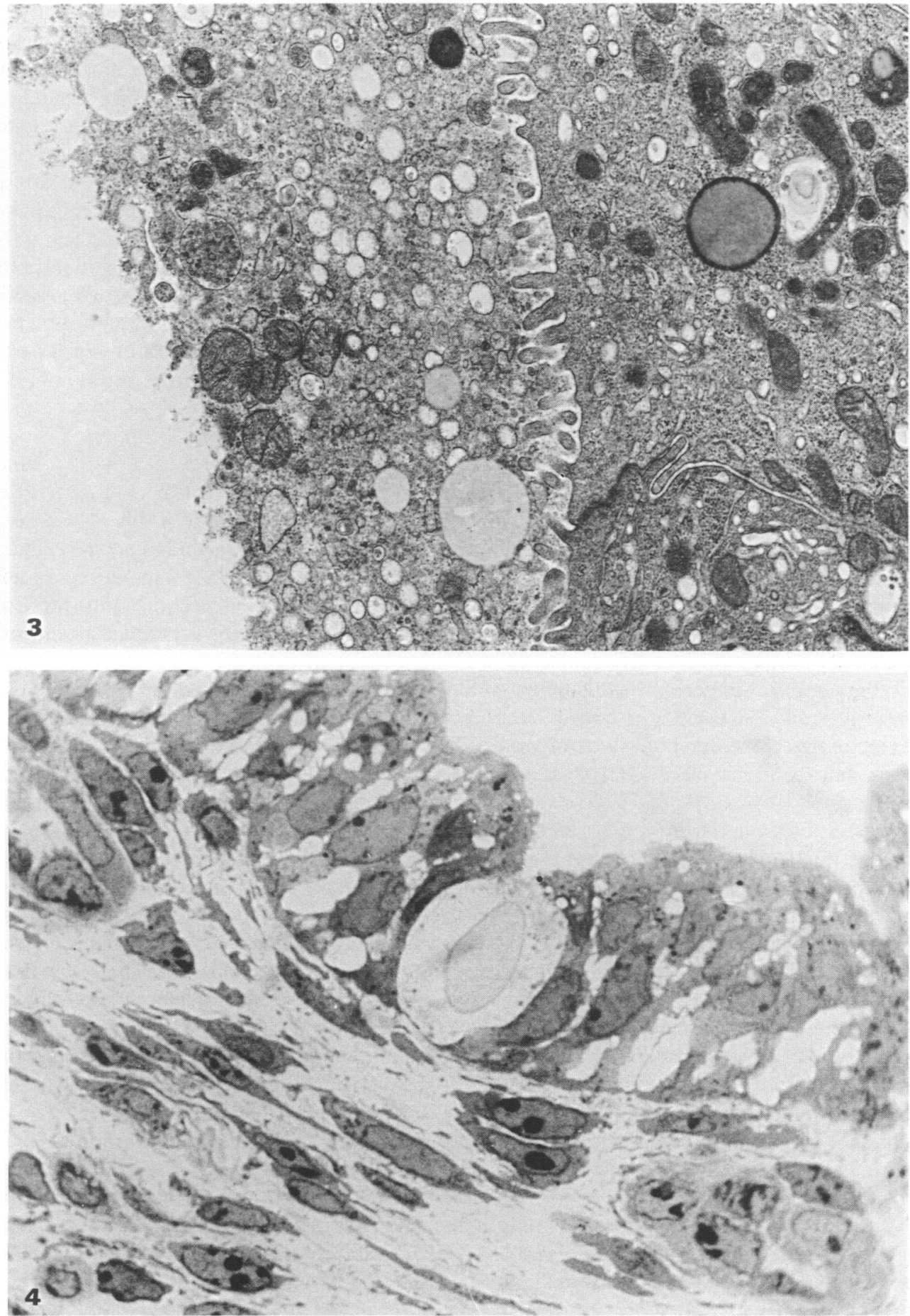

Fig. 3. Electron micrograph of the surface of an epithelial cell from a mouse killed $45 \mathrm{~min}$ after instillation of oil, showing cellular debris in the lumen. $\times 16000$.

Fig. 4. Light micrograph of the luminal epithelium from a mouse killed within $1 \mathrm{~min}$ of the intrauterine injection of a solution of carrageenan, showing one enlarged pale cell and the lacelike appearance of the epithelium. $\times 400$. 
$(\mathrm{N}=5$ ) later. Control mice $(\mathrm{N}=10)$ received no oestradiol. All mice continued to receive daily injections of $500 \mu \mathrm{g}$ progesterone and 3 days later the uteri were weighed and examined macroscopically and histologically for the presence of decidual tissue. None of the control mice showed macroscopic decidual responses (mean \pm s.e.m. weight of both uterine horns $=106 \pm 4.9 \mathrm{mg}$ ). In contrast, all the animals injected with oestradiol 10 or $16 \mathrm{~h}$ after the oil showed marked decidual responses (uterine weights of $182 \pm 27 \cdot 1$ and $151 \pm 15 \cdot 9 \mathrm{mg}$, respectively).

In the second experiment, oil was instilled into just one uterine horn of mice on the 3rd day of progesterone treatment ( $1 \mathrm{mg}$ daily). The mice received either no oestradiol-17 $\beta(\mathrm{N}=8)$, or $10 \mathrm{ng}$ oestradiol-17 $\beta$ (i) $4 \mathrm{~h}$ before, (ii) $24 \mathrm{~h}$ after or (iii) $48 \mathrm{~h}$ after the oil instillation. The mice continued to receive daily injections of $1 \mathrm{mg}$ progesterone and were killed 2-4 days after the oil instillation for assessment of the decidual response. The results are presented in Table 1. In the absence of oestradiol, no decidual responses were obtained other than at the immediate site of instillation. Extensive decidual responses were obtained in the majority of animals in all the treatments in which oestradiol was given, even when the oestradiol was delayed for $48 \mathrm{~h}$ after the initial intraluminal oil instillation.

\section{Discussion}

It is clear from these results that the intraluminal injection of oil or carrageenan into the uterus of the mouse causes very rapid disruptive changes in some of the cells of the luminal epithelium. Although both of these substances are potent deciduogenic stimuli only in the fully sensitized uterus, they induced the structural changes regardless of whether or not the uterus was hormonally sensitized for implantation or decidualization. This is consistent with the fact that oil instillation also induces rapid changes in prostaglandin concentrations (Milligan \& Lytton, 1983) and vascular permeability (Milligan, 1987) in both sensitized and non-sensitized uteri. No structural changes were seen in the epithelium after the instillation of saline in the present study. Saline is not deciduogenic in mice and its instillation does not induce any marked increase in uterine vascular permeability (Milligan, 1987). The present results are not consistent with the observations of Lundkvist \& Nilsson (1982) in rats. Although these authors also observed rapid (within $15 \mathrm{~min}$ ) damage to the luminal epithelium after the instillation of artificial deciduogenic stimuli (oil, air), in their experiments the damage only occurred in uteri which had been maximally sensitized with the appropriate combination of progesterone and oestradiol.

The differences between the epithelial damage caused by oil and by carrageenan in mice in the present study were unexpected. Although the end result, that is the loss of cells from the epithelium, was the same for both stimuli, the method by which this was achieved was different. With oil, the cellular contents appeared to be extruded into the lumen. Similar observations were made by Lundkvist \& Nilsson (1982) for the rat. With carrageenan, however, this does not occur and the route of cell loss is uncertain. In both cases, the integrity of the epithelium is rapidly re-established.

Whether the epithelial disruption induced in the epithelium by artificial stimuli acts as the stimulus for decidualization is uncertain. While gross uterine trauma (e.g. crushing, scratching) readily induces decidualization in non-sensitized (progesterone-only treated) mice, the epithelial damage induced by oil or carrageenan is only effective in inducing decidualization in combination with a sensitizing injection of oestradiol (Finn, 1965). This indicates that the early structural effects of such intrauterine stimuli on the epithelium do not provide a sufficient stimulus in non-sensitized uteri. Possibly there are differences between oestrogen-sensitized and non-sensitized uteri, either in the ability of the luminal epithelial cells to respond to the decidualizing stimulus, or in the sensitivity or potential of the underlying stromal cells to respond to such signals that are produced.

The time course of the epithelial damage is also interesting in relation to the ability of oil to induce decidualization even when the sensitizing oestradiol was given 10 or $16 \mathrm{~h}$ after the oil, well 


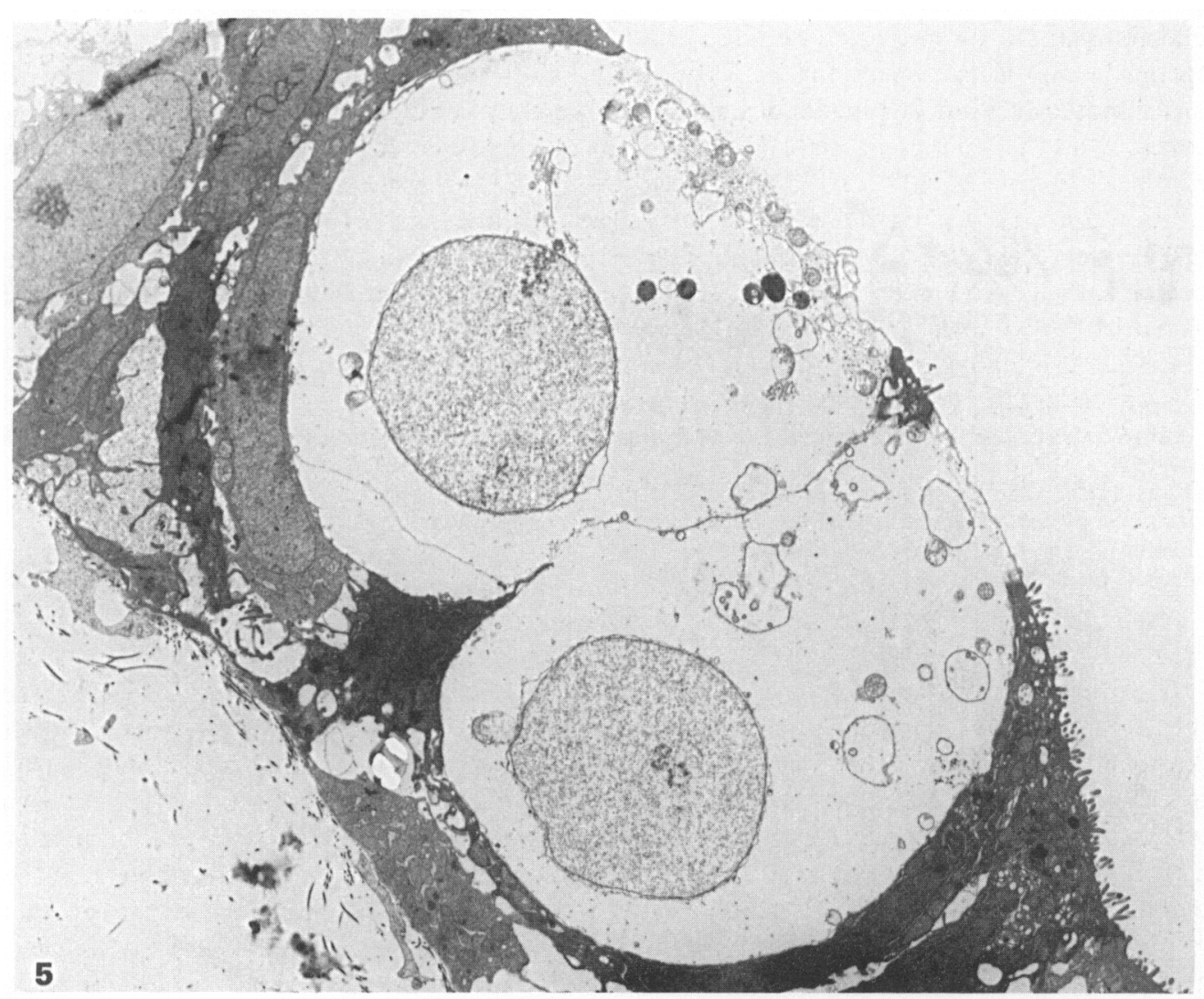

Fig. 5. Electron micrograph of two pale cells in the uterine epithelium of a mouse killed within 1 min of injection of carrageenan. $\times 4600$.

Table 1. Uterine decidual cell responses in progesterone-treated ( $1 \mathrm{mg}$ daily) mice in relation to the interval between the intraluminal instillation of oil and the nidatory oestradiol $(10 \mathrm{ng})$

\begin{tabular}{|c|c|c|c|c|}
\hline \multirow{2}{*}{$\begin{array}{l}\text { Time of } \\
\text { oestradiol } \\
\text { injection }\end{array}$} & \multirow{2}{*}{$\begin{array}{c}\text { Interval (days) } \\
\text { between oestradiol } \\
\text { and death }\end{array}$} & \multirow{2}{*}{$\begin{array}{l}\text { No. of } \\
\text { mice }\end{array}$} & \multicolumn{2}{|c|}{ Uterine weight (mg) } \\
\hline & & & Control horn & Instilled horn \\
\hline & 2 & 8 & $25 \cdot 1 \pm 2 \cdot 8$ & $40 \cdot 6 \pm 3 \cdot 8$ \\
\hline $4 \mathrm{~h}$ before oil* & 2 & 8 & $27 \cdot 0 \pm 4 \cdot 6$ & $137 \pm 14 \cdot 8$ \\
\hline $24 \mathrm{~h}$ after oil & 4 & 12 & $20 \cdot 0 \pm 2 \cdot 2 \dagger$ & $106 \pm 18 \cdot 7$ \\
\hline $48 \mathrm{~h}$ after oil & 3 & 12 & $17 \cdot 0 \pm 1 \cdot 0 \ddagger$ & $90 \cdot 3 \pm 15 \cdot 5$ \\
\hline
\end{tabular}

*Instilled into one horn on 3rd day of progesterone treatment.

Omitting data from $2 \dagger$ and $3 \ddagger$ control horns with decidual responses.

Values are mean \pm s.e.m.

after any signs of trauma have disappeared from the uterus. This indicates that the small oil droplets remaining in the uteri at this time are still capable of acting as effective decidualizing stimuli, even though the structural damage to the epithelium has been repaired. It suggests that the early structural damage is unrelated to the decidualizing stimulus given by the oil. Whatever the stimulus 
is, it appears to operate on the luminal surface of the intact epithelial cells, in a similar manner to the blastocyst.

We thank Mrs Audrey Meade for help in the preparation of the manuscript.

\section{References}

Finn, C.A. (1965) Oestrogen and the decidual cell reaction of implantation in mice. J. Endocr. 32, 223-229.

Finn, C.A. \& Keen, P.M. (1963) The induction of deciduomata in the rat. J. Embryol. exp. Morph. 11, 673-682.

Finn, C.A. \& Martin, L. (1969) Hormone secretion during early pregnancy in the mouse. $J$. Endocr. 45, $57-65$.

Loeb, L. (1908) The experimental production of the maternal placenta and the formation of the corpus luteum. J. Am. med. Ass. 50, 1897-1901.

Lundkvist, O. \& Nilsson, O. (1982) Endometrial ultra- structure in the early uterine response to blastocysts and artificial deciduogenic stimuli in rats. Cell Tiss. Res. 225, 355-364.

Milligan, S.R. (1987) The sensitivity of the uterus of the mouse and rat to intraluminal instillation. $J$. Reprod. Fert. 79, 251-259.

Milligan, S.R. \& Lytton, F.D.C. (1983) Changes in prostaglandin levels in the sensitized and non-sensitized uterus of the mouse after the intrauterine instillation of oil or saline. J. Reprod. Fert. 67, 373-377.

Received 7 November 1988 